\title{
Prevalencia y factores de virulencia de Staphylococcus coagulasa negativos causantes de infección de prótesis articular en un hospital ortopédico de México
}

\author{
Prevalence and virulence factors of coagulase negative Staphylococcus causative of \\ prosthetic joint infections in an orthopedic hospital of Mexico
}

\author{
Silvestre Ortega-Peña ${ }^{1,2}$, Rafael Franco-Cendejas', Brenda Salazar-Sáenz¹, Sandra Rodríguez-Martínez², \\ Mario E. Cancino-Díaz y Juan C. Cancino-Díaz ${ }^{2 *}$ \\ ${ }^{1}$ Laboratorio de Infectología, Instituto Nacional de Rehabilitación Luis Guillermo Ibarra Ibarra; 'Departamento de Microbiología e Inmunología, \\ Escuela Nacional de Ciencias Biológicas, Instituto Politécnico Nacional. Ciudad de México, México
}

\section{Resumen}

Objetivo: Estudiar la prevalencia y los factores de virulencia de Staphylococcus coagulasa negativos (SCN) de infecciones de prótesis articular (IPA). Método: Los SCN se aislaron de 66 pacientes con IPA de cadera y rodilla procedentes del Instituto Nacional de Rehabilitación Luis Guillermo Ibarra Ibarra, de Ciudad de México. Se determinaron la sensibilidad antimicrobiana y la producción de biopelículas de los SCN. Los genes icaADBC, aap, bap y embp fueron detectados por reacción en cadena de la polimerasa en SCN. Resultados: La IPA de cadera fue el 80\%. Se aislaron en alta proporción S. epidermidis (82\%) y S. hominis (80\%), y en baja frecuencia S. lugdunensis, S. haemolyticus, S. capitis, S. caprae, S. sciuri y $S$. lentus. La mayoría de los aislamientos fueron resistentes a los betalactámicos, las fluoroquinolonas y la eritromicina. La producción de biopelículas se determinó en el 41\% de los SCN y el 59\% fueron no productores de biopelículas ( $p=0.0551)$. $S$. epidermidis productores de biopelículas tuvieron mayor presencia del operón icaADBC, aap y embp que los aislamientos no productores de biopelícula $(p<0.05)$. Los SCN no $S$. epidermidis presentaron únicamente el gen aap. Conclusiones: $S$. haemolyticus, $S$. sciuri y $S$. lentus son aislamientos nuevos de IPA no reportados que poseen factores de virulencia, igual que las otras especies de SCN aisladas.

PALABRAS CLAVE: Coagulasa negativa. Prótesis articular. Staphylococcus. Virulencia. Prevalencia.

\begin{abstract}
Objective: To determine the prevalence and virulence factors of coagulase-negative Staphylococci (CNS) in prosthetic joint infections (PJI). Method: CNS were isolated of 66 hip and knee PJI from Instituto Nacional de Rehabilitación Luis Guillermo Ibarra Ibarra, México City. Antimicrobial susceptibility and biofilm formation in CNS were determined; icaADBC, aap, bap and embp genes were determined by PCR. Results: Staphylococcus and Staphylococcus hominis were the most prevalent with 82 y $80 \%$ respectively. Staphylococcus lugdunensis, Staphylococcus haemolyticus, Staphylococcus capitis, Staphylococcus caprae, Staphylococcus sciuri and Staphylococcus lentus were less frequent. The majority of isolates were resistant to $\beta$-lactam antibiotics, fluoroquinolone, and erythromycin. $41 \%$ of CNS were biofilm former and 59\% were non-biofilm former
\end{abstract}

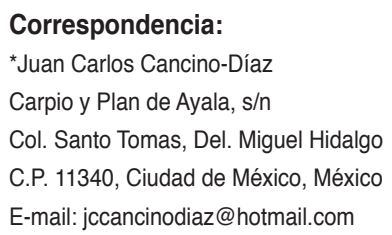

Fecha de recepción: 01-09-2018

Fecha de aceptación: 05-02-2019

DOI: $10.24875 / C I R U .19000690$
Cir Cir. 2019;87:428-435

Contents available at PubMed www.cirugiaycirujanos.com 
$(p=0.0551)$. Biofilm former Staphylococcus epidermidis showed a high presence of icaADBC, aap and embp operons compared to the non-biofilm former isolates $(p<0.05)$. In contrast, non-S. epidermidis CNS had only the aap gen. Conclusion: S. haemolyticus, $S$. sciuri and $S$. lentus are new isolates of $P J I$ not previously reported with virulence factors similar to CNS isolates.

KEY WORDS: Coagulase negative. Prevalence. Prosthetic joint. Staphylococcus. Virulence.

\section{Introducción}

Las infecciones de prótesis articular (IPA) de cadera y rodilla son las más temidas, debido al tratamiento complicado'; además, los lavados quirúrgicos frecuentes y las estancias hospitalarias prolongadas ${ }^{1,2}$ provocan elevados costos económicos para el sistema de salud y para los propios pacientes. La incidencia de IPA es del $1.9 \%$ en las artroplastias primarias de cadera y del $2.1 \%$ en las de rodilla, y del $30-40 \%$ en las artroplastias de revisión tanto de cadera como de rodilla'. Las IPA afectan principalmente a personas de la tercera edad ${ }^{1}$. La causa más común de IPA es la contaminación del material protésico con microorganismos, y la vía de contaminación puede ser hematógena o por inoculación directa durante el procedimiento quirúrgico ${ }^{3}$.

Los agentes etiológicos más comunes de IPA son los miembros del género Staphylococcus, y dentro de él las especies que más se aíslan son Staphylococcus aureus y Staphylococcus epidermidis ${ }^{4}$. En la actualidad se aíslan, en menor proporción, otras especies de Staphylococcus coagulasa negativos (SCN) no $S$. epidermidis causantes de IPA. Los SCN no habían sido importantes en las IPA debido a su bajo perfil de virulencia ${ }^{5-7}$, pero hoy en día se ha demostrado su capacidad infectiva.

En los últimos años, los SCN no S. epidermidis han adquirido gran relevancia debido a que se han convertido en patógenos oportunistas importantes, principalmente en infecciones asociadas al uso de implantes médicos, como las prótesis articulares ${ }^{8}$. En general, los SCN causan infecciones crónicas, persistentes y difíciles de tratar con antimicrobianos convencionales, y esto se ha atribuido a que la mayoría de las especies son reservorios naturales de genes de resistencia antimicrobiana y a su habilidad para producir biopelículas. Estas son el principal factor de virulencia de los SCN, ya que las células bacterianas dentro de la biopelícula están protegidas de los componentes de la respuesta inmunitaria del huésped y de las moléculas con actividad antimicrobiana ${ }^{8,9}$.
El mecanismo molecular de la formación de biopelículas ha sido estudiado ampliamente en $S$. epidermidis ${ }^{10}$. Hasta la fecha, se sabe que algunas cepas de $S$. epidermidis pueden formar biopelículas por dos mecanismos. El primero es por la presencia del operón ica, el cual participa en la síntesis del polisacárido de adhesión intercelular (PAI) ${ }^{11}$. Este operón está constituido por los genes icaA, icaD, icaB, icaC e $i c a R^{11}$. El PAl es el exopolisacárido que envuelve a las bacterias y las protege de los compuestos antimicrobianos y de la respuesta inmunitaria del huésped. El segundo mecanismo descrito es independiente de PAl, y el componente principal de las biopelículas es la proteína extracelular. En este mecanismo participan la proteína asociada a la adhesión (Aap, codificada por el gen aap), la proteína asociada a biopelícula (codificada por el gen bap) y la proteína de unión a la matriz extracelular asociada a biopelícula (codificada por el gen embp $)^{12}$. Esta biopelícula independiente de PAI es más débil que la biopelícula conformada por PAI. Sin embargo, se ha determinado que las cepas de $S$. epidermidis que producen biopelículas de tipo PAI o biopelículas independientes de PAI son virulen$\operatorname{tas}^{13}$. No todos los aislamientos clínicos o comensales de SCN tienen la capacidad de producir biopelículas; diversos estudios han documentado que la producción de biopelículas puede variar entre las especies de Staphylococcus, inclusive entre cepas de una misma especie, y esto se ha atribuido a la presencia o ausencia de los genes asociados a biopelículas (operón ica y genes aap, bap y embp) $)^{8,9,14}$.

Se ha estudiado la prevalencia de los genes asociados a biopelículas en $S$. epidermidis aislados de IPA en hospitales ortopédicos. Estos estudios han reportado que hay una correlación entre la presencia de los genes asociados a biopelículas y la IPA. Tales hallazgos han permitido entender la patogénesis de los SCN de IPA, así como identificar posibles dianas terapéuticas que funcionen de manera diferente al uso de los antimicrobianos (por ejemplo, vacunas) para la prevención de la IPA ${ }^{15-17}$. En México no hay estudios de prevalencia de los genes asociados a biopelículas de SCN en IPA; solo se dispone de 
estudios sobre SCN aislados de otro tipo de infecciones, como oculares, de catéteres intravasculares, bacteriemias, etc. ${ }^{11}$. Por lo tanto, el objetivo de este estudio fue describir la prevalencia de SCN en IPA, así como las características de virulencia de las cepas productoras de biopelículas y los genes asociados a ella.

\section{Métodos}

\section{Población de estudio}

La base de datos electrónica del laboratorio de infectología fue revisada retrospectivamente para identificar y recuperar la información demográfica, clínica y microbiológica de los pacientes que fueron diagnosticados de IPA (cadera y rodilla) causada por SCN, entre enero de 2011 y diciembre de 2015. Este es un estudio retrospectivo a partir de una base de datos, y por tal motivo no fue necesaria la aprobación de comité de ética e investigación.

\section{Cepas}

Los aislamientos clínicos de SCN fueron cultivados a partir de muestras de tejidos que estuvieron en contacto con la prótesis articular, el tejido óseo, el tejido periprotésico, los fluidos articulares y el líquido de sonicación de la prótesis articular (cuando en algunos casos fue retirada). La identificación y el perfil de sensibilidad antimicrobiana (varias familias de antibióticos) de los aislamientos se realizaron usando el sistema semiautomatizado Vitek 2. Todos los aislamientos estuvieron almacenados en caldo MüeIler-Hinton suplementado con glicerol al $25 \%$ (v/v) a $-70{ }^{\circ} \mathrm{C}$ hasta que fueron utilizados para su análisis.

\section{Determinación in vitro de la formación de biopelículas}

La formación de biopelículas fue evaluada por el ensayo de tinción con cristal violeta. Los aislamientos crecieron toda una noche en caldo de soya tripticaseína (CST). Enseguida se diluyeron 1:100 en CST suplementado con glucosa al $1 \%(\mathrm{p} / \mathrm{v})$. Cada dilución fue depositada en tres pocillos de fondo plano de una microplaca estéril de 96 pozos (Falcon, Frankling Lakes, NJ). Después de la incubación durante 24 horas a $37^{\circ} \mathrm{C}$, el caldo fue eliminado y los pocillos fueron lavados vigorosamente (dos veces) con regulados de fosfatos $1 \mathrm{X}$ (PBS), secados por 30 minutos a $55^{\circ} \mathrm{C}$ y teñidos con una solución de cristal violeta al $0.5 \%(\mathrm{p} / \mathrm{v})$ por 15 minutos. Se eliminó el colorante y el exceso fue eliminado lavando nuevamente los pocillos con PBS 1X. El colorante retenido en las biopelículas se extrajo con $200 \mathrm{ml}$ de etanol al 30\%, y la absorbancia (As) se cuantificó a $595 \mathrm{~nm}$ en un espectrofotómetro Multiskan EX Microplate Photometer (Thermo Scientific). La formación de biopelículas se evaluó usando el punto de corte sugerido por Christensen, et al. ${ }^{18}$, aquellas $\mathrm{As}_{595 \mathrm{~nm}} \leq 0.120$, los aislamientos se clasificaron como no productores de biopelículas. Si $\mathrm{As}_{595 \mathrm{~nm}}>0.240$, los aislamientos se clasificaron como productores de biopelículas de nivel fuerte. Los aislamientos con $\mathrm{As}_{595 \mathrm{~nm}}>0.120 \mathrm{y} \leq 0.240$ se clasificaron como productores de biopelículas de nivel débil. El ensayo se repitió dos veces en experimentos independientes y se usaron los valores medios de la absorbancia de biopelículas. Los resultados fueron analizados usando una ANOVA de una vía con prueba Tukey.

\section{Extracción de DNA genómico}

Las células bacterianas crecieron toda una noche en CST y luego fueron cosechadas por centrifugación y resuspendidas en $200 \mathrm{ml}$ de una solución de lisis (sacarosa al $20 \%$, Tris- $\mathrm{HCl} 10 \mathrm{mM} \mathrm{pH} 8$ y lisozima $10 \mathrm{mg} / \mathrm{ml}$ ). Enseguida se incubaron a $37^{\circ} \mathrm{C}$ por $40 \mathrm{mi}-$ nutos con $200 \mathrm{ml}$ de solución Whinston (triton $\mathrm{X}-1002 \%$, SDS 1\%, $\mathrm{NaCl} 10 \mathrm{nM}$, Tris base $10 \mathrm{mM}$, pH 8 y EDTA $1 \mathrm{mM}$ ). El DNA fue extraído con la técnica de fenol-cloroformo. Finalmente, el DNA se resuspendió en agua destilada estéril.

\section{Amplificación de genes asociados a biopelículas y gen mecA (resistencia a oxacilina)}

El DNA genómico de los aislamientos fue usado como molde para la amplificación del operón icaADBC y los genes embp, bap, aap y mecA con los iniciadores descritos previamente ${ }^{19}$. Las reacciones se hicieron con $1 \mu \mathrm{l}$ de DNA (100 ng), amortiguador de la reacción en cadena de la polimerasa (PCR) $1 \mathrm{X}, \mathrm{MgCl}_{2} 1 \mathrm{mM}$, dNTPs $200 \mu \mathrm{M}$ cada uno, $1 \mathrm{U}$ de Taq DNA polimerasa (Invitrogen, Carlsbad, CA, USA) y $0.2 \mu \mathrm{M}$ de cada iniciador específico. Las condiciones óptimas de PCR fueron 30 ciclos por 30 segundos a $92^{\circ} \mathrm{C}$, 40 segundos a $60^{\circ} \mathrm{C}$ y 30 segundos a $72{ }^{\circ} \mathrm{C}$. Los productos de PCR se analizaron en geles de agarosa al $1 \%(p / v)$. EI DNA genómico de S. epidermidis ATCC35984 fue 
Tabla 1. Tipo de prótesis infectada, estafilococo coagulasa negativo identificado y perfil de sensibilidad antimicrobiana

\begin{tabular}{|c|c|c|c|c|c|c|c|c|c|c|c|c|c|c|}
\hline \multirow{2}{*}{$\begin{array}{l}\text { Aislamientos } \\
\mathrm{n}(\%)\end{array}$} & \multirow{2}{*}{$\begin{array}{c}\text { Cadera } \\
\text { n (\%) }\end{array}$} & \multirow{2}{*}{$\begin{array}{c}\text { Rodilla } \\
\text { n (\%) }\end{array}$} & \multicolumn{12}{|c|}{ Perfil de resistencia antimicrobiana $\mathrm{n}(\%)$} \\
\hline & & & $\mathbf{P}$ & ox & $\mathrm{Cl}$ & LE & MXF & $\mathbf{E}$ & $\mathrm{CC}$ & LZ & VA & TE & $\mathbf{R} \mathbf{I}$ & SXT \\
\hline S. epidermidis, 55 (85) & $45(82)$ & $10(18)$ & $44(80)$ & $43(78)$ & $30(55)$ & $32(58)$ & $33(75)$ & $19(34)$ & $12(27)$ & 0 & 0 & $10(23)$ & $6(14)$ & $22(50)$ \\
\hline S. hominis, 5 (9) & $4(80)$ & $1(20)$ & $5(100)$ & $5(100)$ & $5(100)$ & $5(100)$ & $5(100)$ & $5(100)$ & 0 & 0 & 0 & 0 & 0 & $5(100)$ \\
\hline S. lugdunensis, 1 (1) & 0 & $1(100)$ & $1(100)$ & $1(100)$ & $1(100)$ & $1(100)$ & $1(100)$ & $1(100)$ & 0 & 0 & 0 & 0 & 0 & 0 \\
\hline S. haemolyticus, 1 (1) & 0 & $1(100)$ & $1(100)$ & $1(100)$ & $1(100)$ & $1(100)$ & $1(100)$ & $1(100)$ & 0 & 0 & 0 & 0 & 0 & 0 \\
\hline S. capitis, 1 (1) & $1(100)$ & 0 & 0 & 0 & 0 & 0 & 0 & 0 & 0 & 0 & 0 & 0 & 0 & 0 \\
\hline S. caprae, 1 (1) & $1(100)$ & 0 & 0 & 0 & 0 & 0 & 0 & 0 & 0 & 0 & 0 & 0 & 0 & 0 \\
\hline S. sciuri, 1 (1) & $1(100)$ & 0 & $1(100)$ & $1(100)$ & 0 & 0 & 0 & 0 & $1(100)$ & 0 & 0 & 0 & 0 & 0 \\
\hline S. lentus, 1 (1) & $1(100)$ & 0 & $1(100)$ & $1(100)$ & $1(100)$ & $1(100)$ & $1(100)$ & $1(100)$ & 0 & 0 & 0 & 0 & 0 & 0 \\
\hline Total & $53(80)$ & $13(20)$ & $53(80)$ & $51(77)$ & $37(56)$ & $38(57)$ & $39(59)$ & $26(39)$ & $15(23)$ & 0 & 0 & $10(15)$ & $6(9)$ & $25(35)$ \\
\hline
\end{tabular}

usado como control positivo para el operón icaADBC y los genes bap, aap y embp. Se utilizó $S$. aureus ATCC 43300 como control positivo para el gen mecA.

\section{Resultados}

\section{Identificación de SCN y sensibilidad antimicrobiana}

En total se identificaron 66 pacientes con IPA causada por SCN. El 65\% $(n=43)$ pertenecían al sexo femenino. La mediana de edad de la población seleccionada fue de 64 años (rango: 19-91). El 99\% $(n=65)$ de los casos de IPA se asociaron a artroplastias de revisión, y en el $73 \%(n=48)$ no se pudo retener la prótesis articular.

En la tabla 1 se muestra el tipo de prótesis infectada, el SCN identificado y el perfil de sensibilidad antimicrobiana. Con respecto al tipo de prótesis infectada, el $80 \%(n=53)$ de los pacientes fueron IPA de cadera y el resto IPA de rodilla $(n=13)$. $S$. epidermidis $(82 \%)$ y $S$. hominis $(80 \%)$ fueron aislados en mayor proporción de pacientes con IPA de cadera que de pacientes con IPA de rodilla. S. Iugdunensis y $S$. haemolyticus fueron aislados únicamente de pacientes con IPA de rodilla. Contrario a esto, S. capitis, $S$. caprae, $S$. sciuri y $S$. lentus fueron aislados de pacientes con IPA de cadera.

Los aislamientos clínicos de $S$. epidermidis y $S$. hominis tuvieron altos porcentajes de resistencia a penicilina, oxacilina, moxifloxacino ciprofloxacino, levofloxacino, trimetoprima-sulfametoxazol y eritromicina (Tabla 1).
S. lugdunensis, S. haemolyticus y S. lentus solo fueron sensibles a clindamicina, linezolid, vancomicina, tetraciclina y rifampicina. $S$. sciuri solo presento resistencia a penicilina, oxacilina y clindamicina. $S$. capitis y $S$. caprae fueron sensibles a todos los antibióticos probados (Tabla 1). Con la finalidad de confirmar la resistencia a oxacilina, el gen mecA fue detectado en todos los aislamientos; todos los aislamientos resistentes a oxacilina $(n=52)$ presentaron el gen mecA en su genoma y aquellos sensibles a oxacilina no tuvieron dicho gen.

\section{Producción de biopelículas y presencia de genes asociados a biopelículas}

Con la finalidad de determinar otros factores de virulencia diferentes a la resistencia antimicrobiana, la producción de biopelículas y los genes asociados a biopelículas (icaADBC, aap, bap y embp) fueron detectados en todos los aislamientos de SCN. La producción de biopelículas se determinó en el $41 \%$ $(n=27)$ de los aislamientos de SCN; el resto de los aislamientos $(59 \%, n=39)$ fueron no productores de biopelículas $(p=0.0551)$. De los aislamientos de SCN productores de biopelículas, solo $S$. epidermidis fue productor de biopelículas de nivel fuerte $(13.6 \%$, $n=9$ ); los otros SNC no $S$. epidermidis fueron productores de biopelículas de nivel débil $(12.1 \%, n=8)$. S. capitis, S. caprae y $S$. sciuri no fueron productores de biopelículas.

Por especies, encontramos que $S$. epidermidis tuvo una alta prevalencia de cepas no productoras de 
Tabla 2. Producción de biopelículas y genes asociados a biopelículas en los aislamientos de estafilococos coagulasa negativos

\begin{tabular}{|c|c|c|c|c|c|}
\hline \multirow[t]{2}{*}{ Especie } & \multirow[b]{2}{*}{$\begin{array}{c}\text { Biopelículas } \\
\text { n (\%) }\end{array}$} & \multicolumn{4}{|c|}{ Genotipo } \\
\hline & & $\begin{array}{c}\text { icaADBC } \\
\mathrm{n}(\%)\end{array}$ & $\begin{array}{c}\text { aap } \\
\mathrm{n}(\%)\end{array}$ & $\begin{array}{c}\text { bap } \\
\text { n (\%) }\end{array}$ & $\begin{array}{l}\text { embp } \\
\mathrm{n}(\%)\end{array}$ \\
\hline \multirow[t]{3}{*}{ S. epidermidis } & N 36 (65.5) & $7(19.4)^{\mathrm{B}}$ & $24(66.6)^{A}$ & $11(30.5)^{A}$ & $16(44.4)^{A}$ \\
\hline & D $9(16.4)$ & $1(11.1)^{\mathrm{B}}$ & $7(77.7)^{\mathrm{A}}$ & $O^{A}$ & $6(66.6)^{A}$ \\
\hline & F $10(18.1)$ & $8(80)^{A}$ & $8(80)^{A}$ & $2(20)^{A}$ & $8(80)^{A}$ \\
\hline Total & 55 & $16(29)$ & $39(71)$ & $13(24)$ & $30(54)$ \\
\hline S. hominis & D $5(100)$ & 0 & $5(100)$ & 0 & 0 \\
\hline S. Iugdunensis & D $1(100)$ & 0 & $1(100)$ & 0 & 0 \\
\hline S. haemolyticus & D $1(100)$ & 0 & $1(100)$ & 0 & 0 \\
\hline S. capitis & N $1(100)$ & 0 & $1(100)$ & 0 & 0 \\
\hline S. caprae & N $1(100)$ & 0 & $1(100)$ & 0 & 0 \\
\hline S. sciuri & N $1(100)$ & 0 & $1(100)$ & 0 & 0 \\
\hline S. lentus & D $1(100)$ & 0 & $1(100)$ & 0 & 0 \\
\hline
\end{tabular}

D: productores de biopelículas de nivel débil; F: productores de biopelículas de nivel fuerte; $\mathrm{N}$ : no productores de biopelículas.

Las letras diferentes (A y B) significan diferencia significativa ( $p<0.05$ ); las letras iguales (A) significan no diferencia significativa. El análisis estadístico se realizó con la prueba exacta de Fisher.

biopelículas $(65.5 \%)$ en comparación con las cepas productoras de biopelículas $(34.5 \% ; p=0.0021)$; de las cepas productoras de biopelícula, el $16 \%$ fueron productoras de biopelículas de nivel fuerte y el $18 \%$ de nivel débil. Entre las especies de SCN no S. epidermidis, S. hominis, S. lugdunensis, S. haemolyticus y $S$. lentus fueron productoras de biopelículas de nivel débil.

Con la finalidad de correlacionar la producción de biopelículas y los genes asociados a biopelículas, estos genes fueron detectados y analizados por especie y por grupos de biopelículas de nivel débil, biopelículas de nivel fuerte y no productoras de biopelículas. Con respecto al grupo de $S$. epidermidis productores de biopelículas de nivel fuerte, el $80 \%$ de los aislamientos tuvieron el operón icaADBC y los genes aap y embp; el grupo de $S$. epidermidis productores de biopelículas de nivel débil presentaron menor proporción del operón icaADBC (11.1\%) y mayor proporción de los genes aap (77.7\%) y embp (66.6\%). De manera similar, en el grupo de $S$. epidermidis no productores de biopelículas se observa una mayor proporción de los genes aap y embp que del operón icaADBC (Tabla 2). Comparando entre los diferentes grupos de $S$. epidermidis se encontró que solo los productores de biopelículas de nivel fuerte fueron significativamente diferentes en cuanto a la presencia del operón icaADBC que el grupo de productores de biopelículas de nivel débil y el grupo de no productores de biopelículas $(p=0.0055)$. En los otros genes determinados no hubo diferencia significativa entre los diferentes grupos (Tabla 2).

Para el caso de los SCN no S. epidermidis, todos los aislamientos productores de biopelículas de nivel débil tuvieron la presencia del gen aap y la ausencia del operón ica $A D B C$ en su genoma, y de igual manera para $S$. capitis, S. caprae y $S$. sciuri (no productores de biopelículas).

\section{Discusión}

Staphylococcus spp. son los microorganismos que más causan IPA, siendo $S$. aureus y $S$. epidermidis las especies que más se aíslan. Sin embargo, en diversos estudios epidemiológicos que describen las características microbiológicas de las IPA se ha reportado, en las últimas décadas, un incremento del aislamiento de otras especies de SCN diferentes a S. epidermidis ${ }^{20-22}$. En nuestro país se han publicado pocos estudios sobre la frecuencia de SCN causantes de IPA. Por ello, en el presente estudio nosotros revisamos 66 casos de pacientes diagnosticados de IPA causadas por SCN durante un periodo de cuatro años (2011 a 2015) en un hospital ortopédico de referencia de México. Nuestros resultados muestran que, durante el periodo de estudio, las especies de SCN que más causaron IPA fueron $S$. epidermidis y $S$. hominis, y en menor proporción S. lugdunensis, S. haemolyticus, 
S. capitis, S. caprae, S. sciuri y S. lentus. Resultados similares fueron reportados por el estudio de Montanaro et al. ${ }^{22}$, quienes analizaron las características microbiológicas de 242 casos de IPA (cadera y rodilla) diagnosticados en un hospital ortopédico de referencia en Italia y encontraron que el $75 \%$ de las IPA fueron causadas por Staphylococcus spp., de los cuales las especies de SCN fueron las que más se aislaron, y entre ellas $S$. epidermidis fue la más común, seguida por S. warneri, S. haemolyticus, S. hominis, S. lugdunensis y $S$. capitis.

Un par de décadas atrás, los SCN no S. epidermidis no eran considerados causantes de procesos infecciosos debido a su baja frecuencia de aislamiento, pero el incremento en el uso de dispositivos médicos (válvulas cardiacas, lentes de contacto, marcapasos, prótesis articulares, etc.) ha provocado un aumento del aislamiento de SCN no $S$. epidermidis causantes de infecciones crónicas, persistentes y difíciles de $\operatorname{tratar}^{5-8}$. Los SCN no $S$. epidermidis identificados como causantes de IPA son $S$. hominis, S. lugdunensis, S. capitis y S. caprae ${ }^{23-28}$. En la colección de SCN que aislamos también identificamos estas mismas especies como causantes de IPA en los pacientes atendidos en nuestro hospital. Sin embargo, hallamos otras especies no reportadas como causantes de IPA, tales como S. haemolyticus, S. sciuri y S. lentus. Estas especies se han aislado de otros procesos infecciosos; por ejemplo, $S$. haemolyticus, junto con $S$. epidermidis, se aíslan con alta prevalencia en infecciones relacionadas con catéteres ${ }^{29,30}$. S. lentus y S. sciuri pertenecen al grupo de los Staphylococcus sciuri, que está integrado por $S$. sciuri, $S$. lentus y $S$. vitulinus; este grupo de microorganismos son patógenos de animales y es muy raro que causen infecciones en humanos. Sin embargo, hay reportes de casos de aislamientos de $S$. lentus y $S$. sciuri en peritonitis, infecciones de heridas, endocarditis, infecciones del tracto urinario, endoftalmitis y enfermedad inflamatoria pélvica ${ }^{31-33}$. Nosotros estamos reportando el aislamiento de estas especies en IPA.

Los factores de virulencia que han sido identificados en SCN son la producción de biopelículas y la resistencia antimicrobiana ${ }^{8,9}$. Con respecto a la producción de biopelículas, los estudios han demostrado que puede variar entre especies e intraespecie ${ }^{34}$. En nuestros aislamientos de $S$. epidermidis encontramos variaciones de producción de biopelículas, e inclusive hubo más casos de aislamientos no productores de biopelículas que de aislamientos productores de biopelículas ( $p=0.0021$ ), y esta fue la única especie que produjo biopelículas de nivel fuerte. Los cinco casos de aislamiento de $S$. hominis fueron más consistentes, ya que todos fueron productores de biopelículas de nivel débil. Como anteriormente se mencionó, en SCN se han descrito dos tipos de biopelículas, las dependientes de PAI y las independientes de PAI (biopelículas de tipo proteico), y estos tipos de biopelículas están relacionadas con la presencia o la ausencia de los genes asociados a biopelículas, que son el operón icaADBC (biopelícula dependiente de PAI) y los genes aap, bap y embp (biopelículas de tipo proteico $)^{8,9}$. Rohde, et al..$^{19}$ determinaron la producción de biopelículas en 52 cepas clínicas de S. epidermidis aisladas de IPA (cadera y rodilla) y encontraron que el $50 \%$ de los aislamientos no producen biopelículas, y estos aislamientos tienen una alta prevalencia de los genes independientes de PAI (aap, bap, y embp), mientras que los aislamientos productores de biopelículas tienen los genes del operón icaADBC. Resultados similares encontramos en nuestro estudio: la mayoría de los aislamientos de S. epidermidis tienen alta prevalencia del gen aap, y para el caso del operón icaADBC fue significativa la prevalencia en los aislamientos productores de biopelículas de nivel fuerte en comparación con los productores de nivel débil y con los no productores de biopelículas $(p=0.0055)$. Los aislamientos productores de biopelículas de nivel fuerte tienen una alta prevalencia del operón icaADBC y los genes aap y embp, sugiriendo biopelículas dependientes de IPA y proteicas. Se ha reportado que las biopelículas de nivel fuerte tienen la presencia del operón icaADBC y del gen aap, sugiriendo que la composición de estas biopelículas es de tipo exopolisacárido y de tipo proteico generando una matriz extracelular con mayor fuerza; en el caso de las biopelículas de nivel débil solo tienen la presencia del gen aap. Nuestros resultados muestran que, en las biopelículas nivel débil, se detecta una alta prevalencia del gen aap en $S$. epidermidis y en los SCN no $S$. epidermidis.

Con respecto a los SCN no $S$. epidermidis, estudios fenotípicos y moleculares de $S$. hominis, S. lugdunensis, S. haemolyticus y $S$. caprae aislados de IPA o de infección de sangre indican que producen biopelículas bajo la presencia y la expresión del operón ica $A$ $D B C^{25,27,30,35}$. Nuestros resultados muestran que S. hominis produce biopelículas de nivel débil con la presencia del gen aap, y de la misma manera para $S$. Iugdunensis, $S$. haemolyticus y $S$. lentus, sugiriendo que las biopelículas son de tipo proteico. S. caprae no produce biopelículas y presenta el gen aap, de 
igual manera que $S$. capitis y S. sciuri; nosotros pensamos que estas bacterias podrían estar utilizando otro mecanismo de infección diferente de la producción de biopelículas (aún no descrito). No hay estudios sobre la producción de biopelículas y los genes asociados a esta en $S$. lentus y $S$. sciuri. Tevell, et al. ${ }^{28}$ analizaron una colección de ocho $S$. capitis aislados de IPA que son no productores de biopelículas. Este resultado soporta lo encontrado en la única cepa de S. capitis aislada.

Los programas de vigilancia de la resistencia antimicrobiana en SCN causantes de infecciones sistémicas han reportado que los aislamientos resistentes a la meticilina no solo presentan resistencia a los antibióticos betalactámicos, sino también a otras familias de antibióticos ${ }^{8,11,36}$. Martínez-Meléndez, et al ${ }^{11}$. determinaron el patrón de resistencia en 83 S. epidermidis, $59 \mathrm{~S}$. haemolyticus y $67 \mathrm{~S}$. hominis causantes de bacteriemias en dos hospitales de México (Guadalajara y Nuevo León), y encontraron que las cepas con resistencia a la meticilina presentaron también altos porcentajes de resistencia a levofloxacino, eritromicina y trimetroprima-sulfametoxazol ${ }^{11,35}$. Por otra parte, Arciola, et al. ${ }^{36}$ determinaron el patrón de resistencia antimicrobiana en 15 especies de SCN (S. hominis, S. haemolyticus, S. capitis, S. Iugdunensis, S. lentus, S. caprae y $S$. sciuri) causantes de IPA en el Instituto Ortopédico Rizzoli de Bolonia (Italia), y encontraron que presentaban elevada resistencia a penicilina, oxacilina, ciprofloxacino y eritromicina. Los resultados obtenidos por Martínez-Meléndez, et a ${ }^{11}$. y Arciola, et al ${ }^{36}$ son similares a los nuestros, con la diferencia de que los aislamientos de $S$. caprae y $S$. capitis no presentaron resistencia a ninguno de los antibióticos probados (Tabla 1).

Finalmente, nosotros describimos la prevalencia de especies clínicas de SCN aisladas de IPA y sus características fenotípicas y genotípicas. La principal fortaleza del estudio es ser uno de los primeros en su tipo que se realiza en México, principalmente en SCN aislados de IPA. Sin embargo, aunque es bajo el número de aislamientos de SCN no S. epidermidis, es importante considerar que el periodo de aislamiento fue de 4 años en un solo hospital de la Ciudad de México, contrario a otros trabajos en los que el periodo es mayor (aproximadamente 10 años) y son multicéntricos. Conocer los mecanismos de virulencia de los patógenos de IPA permitirá obtener un conocimiento que pueda utilizarse para la búsqueda de opciones terapéuticas para prevenir o erradicar infecciones causadas por estos patógenos, y que los pacientes que las sufren no presenten efectos secundarios graves.

\section{Conclusiones}

Los resultados de este estudio proporcionan información sobre la prevalencia, las características fenotípicas y genotípicas, y el perfil de sensibilidad antimicrobiana de SCN aislados de IPA. Aunque no se analizó un mayor número de SCN no $S$. epidermidis, los resultados muestran un panorama general de estos patógenos de IPA en la Ciudad de México.

\section{Agradecimientos}

Este trabajo fue apoyado por la Secretaría de Investigación y Posgrado del Instituto Politécnico Nacional, del proyecto con clave 20181167. M. Cancino-Díaz, S. Rodríguez-Martínez y J.C. Cancino-Díaz agradecen las becas COFAA y EDI-IPN, y SNI-CONACyT.

\section{Conflicto de intereses}

Los autores de este trabajo no tienen ningún conflicto de intereses.

\section{Responsabilidades éticas}

Protección de personas y animales. Los autores declaran que para esta investigación no se han realizado experimentos en seres humanos ni en animales.

Confidencialidad de los datos. Los autores declaran que en este artículo no aparecen datos de pacientes.

Derecho a la privacidad y consentimiento informado. Los autores declaran que en este artículo no aparecen datos de pacientes.

\section{Bibliografía}

1. Chen AF, Heller S, Parvizi J. Prosthetic joint infections. Surg Clin North Am. 2014;94:1265-81.

2. Tande AJ, Patel R. Prosthetic joint infection. Clin Microbiol Rev. 2014;27:302-45.

3. Abad CL, Haleem A. Prosthetic joint infections: an update. Curr Infect Dis Rep. 2018;20:15.

4. Puchner SE, Döring K, Staats K, Böhler C, Lass R, Hirschl AM, et al. Sonication culture improves microbiological diagnosis of modular megaprostheses. J Orthop Res. 2017;35:1383-7.

5. Gundtoft PH, Pedersen AB, Schønheyder HC, Møller JK, Overgaard S. One-year incidence of prosthetic joint infection in total hip arthroplasty: a cohort study with linkage of the Danish Hip Arthroplasty Register and Danish Microbiology Databases. Osteoarthritis Cartilage. 2017;25:685-93.

6. Ortega-Peña S, Colín-Castro C, Hernández-Duran M, López-Jácome E, Franco-Cendejas R. Microbiological characteristics and patterns of resistance in prosthetic joint infections in a referral hospital. Cir Cir. 2015;83:371-7.

7. Franco-Cendejas R, Contreras-Córdova EL, Mondragón-Eguiluz JA, Vanegas-Rodríguez ES, Ilizaliturri-Sánchez VM, Galindo-Fraga A. Inci- 


\section{S. Ortega-Peña, et al.: Staphylococcus coagulasa negativos en infección de prótesis articular}

dence of hip and knee prosthetic infections in a specialized center of Mexico City. Cir Cir. 2017;85:485-92.

8. Becker K, Heilmann C, Peters G. Coagulase-negative staphylococci. Clin Microbiol Rev. 2014;27:870-926

9. Ortega-Peña S, Hernández-Zamora E. Microbial biofilms and their impact on medical areas: physiopathology, diagnosis and treatment. Bol Med Hosp Infant Mex. 2018;75:79-88.

10. Büttner H, Mack D, Rohde H. Structural basis of Staphylococcus epidermidis biofilm formation: mechanisms and molecular interactions. Front Cell Infect Microbiol. 2015;5:14.

11. Martínez-Meléndez A, Morfín-Otero R, Villarreal-Treviño L, Camacho-Ortiz A, González-González G, Llaca-Díaz J, et al. Molecular epidemiology of coagulase-negative bloodstream isolates: detection of Staphylococcus epidermidis ST2, ST7 and linezolid-resistant ST23. Braz J Infect Dis. 2016;20:419-28.

12. Speziale P, Pietrocola G, Foster TJ, Geoghegan JA. Protein-based biofilm matrices in Staphylococci. Front Cell Infect Microbiol. 2014;4:171.

13. Post V, Harris LG, Morgenstern M, Mageiros L, Hitchings MD, Méric G, et al. Comparative genomics study of Staphylococcus epidermidis isolates from orthopedic-device-related infections correlated with patient outcome. J Clin Microbiol. 2017;55:3089-103.

14. Giormezis N, Kolonitsiou F, Foka A, Drougka E, Liakopoulos A, Makri A, et al. Coagulase-negative staphylococcal bloodstream and prosthetic-device-associated infections: the role of biofilm formation and distribution of adhesin and toxin genes. J Med Microbiol. 2014;63:1500-8.

15. Hellmark B, Söderquist B, Unemo M, Nilsdotter-Augustinsson A. Comparison of Staphylococcus epidermidis isolated from prosthetic joint infections and commensal isolates in regard to antibiotic susceptibility, agr type, biofilm production, and epidemiology. Int J Med Microbiol. 2013;303:32-9.

16. Månsson $\mathrm{E}$, Hellmark $B$, Sundqvist $M$, Söderquist $B$. Sequence types of Staphylococcus epidermidis associated with prosthetic joint infections are not present in the laminar airflow during prosthetic joint surgery. APMIS. 2015:123:589-95

17. Van Mellaert L, Shahrooei M, Hofmans D, Eldere JV. Immunoprophylaxis and immunotherapy of Staphylococcus epidermidis infections: challenges and prospects. Expert Rev Vaccines. 2012;11:319-34.

18. Christensen GD, Simpson WA, Younger JJ, Baddour LM, Barrett FF, Melton DM, et al. Adherence of coagulase negative staphylococci to plastic tissue culture plates: a quantitative model for the adherence of staphylococci to medical devices. J Clin Microbiol. 1985;22:996-1006.

19. Rohde H, Burandt EC, Siemssen N, Frommelt L, Burdelski C, Wurster S, et al. Polysaccharide intercellular adhesin or protein factors in biofilm accumulation of Staphylococcus epidermidis and Staphylococcus aureus isolated from prosthetic hip and knee joint infections. Biomaterials. 2007;28:1711-20.

20. Holleyman RJ, Baker P, Charlett A, Gould K, Deehan DJ. Microorganis$\mathrm{ms}$ responsible for periprosthetic knee infections in England and Wales. Knee Surg Sports Traumatol Arthrosc. 2016;24:3080-7.

21. Holleyman RJ, Baker PN, Charlett A, Gould K, Deehan DJ. Analysis of causative microorganism in 248 primary hip arthroplasties revised for infection: a study using the NJR dataset. Hip Int. 2016;26:82-9.
22. Montanaro L, Speziale P, Campoccia D, Ravaioli S, Cangini I, Pietrocola G, et al. Scenery of Staphylococcus implant infections in orthopedics. Future Microbiol. 2011;6:1329-49.

23. Szczuka E, Krzymińska S, Bogucka N, Kaznowski A. Multifactorial mechanisms of the pathogenesis of methicillin-resistant Staphylococcus hominis isolated from bloodstream infections. Antonie Van Leeuwenhoek. 2018;111:1259-65.

24. Shah NB, Osmon DR, Fadel H, Patel R, Kohner PC, Steckelberg JM, et al. Laboratory and clinical characteristics of Staphylococcus lugdunensis prosthetic joint infections. J Clin Microbiol. 2010;48:1600-3.

25. Lourtet-Hascoët J, Bicart-See A, Félicé MP, Giordano G, Bonnet E. Staphylococcus lugdunensis, a serious pathogen in periprosthetic joint infections: comparison to Staphylococcus aureus and Staphylococcus epidermidis. Int J Infect Dis. 2016;51:56-61.

26. Seng $P$, Barbe M, Pinelli PO, Gouriet F, Drancourt M, Minebois A et al. Staphylococcus caprae bone and joint infections: a re-emerging infection? Clin Microbiol Infect. 2014;20:1052-8.

27. D'Ersu J, Aubin GG, Mercier P, Nicollet P, Bémer P, Corvec S. Characterization of Staphylococcus caprae clinical isolates involved in human bone and joint infections, compared with goat mastitis isolates. J Clin Microbiol. 2016;54:106-13.

28. Tevell S, Hellmark B, Nilsdotter-Augustinsson Å, Söderquist B. Staphylococcus capitis isolated from prosthetic joint infections. Eur J Clin Microbiol Infect Dis. 2017;36:115-22.

29. Czekaj T, Ciszewski M, Szewczyk EM. Staphylococcus haemolyticus - an emerging threat in the twilight of the antibiotics age. Microbiology. 2015;161:2061-8.

30. Pinheiro L, Brito Cl, Oliveira Ad, Pereira VC, Cunha M de L. Staphylococcus epidermidis and Staphylococcus haemolyticus: detection of biofilm genes and biofilm formation in blood culture isolates from patients in a Brazilian teaching hospital. Diagn Microbiol Infect Dis. 2016;86:11-4.

31. Nemeghaire S, Argudín MA, Feßler AT, Hauschild T, Schwarz S, Butaye $P$. The ecological importance of the Staphylococcus sciuri species group as a reservoir for resistance and virulence genes. Vet Microbiol. 2014;171:342-56.

32. Rivera M, Domínguez MD, Mendiola NR, Roso GR, Quereda C. Staphylococcus lentus peritonitis: a case report. Perit Dial Int. 2014;34:469-70.

33. Cirkovic I, Trajkovic J, Hauschild T, Andersen PS, Shittu A, Larsen AR. Nasal and pharyngeal carriage of methicillin-resistant Staphylococcus sciuri among hospitalised patients and healthcare workers in a Serbian university hospital. PLoS One. 2017;12:e0185181.

34. Sanchez CJ Jr, Mende K, Beckius ML, Akers KS, Romano DR, Wenke JC, et al. Biofilm formation by clinical isolates and the implications in chronic infections. BMC Infect Dis. 2013;13:47.

35. Mendoza-Olazarán S, Morfín-Otero R, Villarreal-Treviño L, Rodríguez-Noriega E, Llaca-Díaz J, Camacho-Ortiz A, et al. Antibiotic susceptibility of biofilm cells and molecular characterisation of Staphylococcus hominis isolates from blood. PLoS One. 2015;10:e0144684.

36. Arciola CR, Campoccia D, An YH, Baldassarri L, Pirini V, Donati ME, et al. Prevalence and antibiotic resistance of 15 minor staphylococcal species colonizing orthopedic implants. Int J Artif Organs. 2006;29:395-401. 